\title{
Biclonal Gammopathies: A Retrospective Study in Hassan II University Hospital Center, Fez, Morocco
}

\author{
Ghammad $\mathrm{W}^{1,2}$, Berrada $\mathrm{S}^{1,2}$, Aissaoui $\mathrm{M}^{2}$, Slaoui $\mathrm{A}^{2}$, Iraqui $\mathrm{FZ}^{2}$, Benbella ${ }^{1,2}$, Mustapha $\mathrm{M}^{1,2}$, El Boukhrissi $\mathrm{F}^{1,3^{*}}$ \\ 1Sidi Mohamed Ben Abdellah University, Faculty of Medicine and Pharmacy, Fez, Morocco \\ 2Biochemistry department, Laboratory of medical analyses, CHU HASSAN II, Fez, Morocco \\ 3Biochemistry and toxicology Laboratory, Military Hospital Moulay Ismail, Meknes, Morocco
}

\begin{abstract}
DOI: $10.36348 /$ sijb.2020.v03i11.001 $\quad$ |Received: 03.11.2020 | Accepted: 14.11.2020 | Published: 20.11 .2020
\end{abstract}
*Corresponding author: El Boukhrissi F

\section{Abstract}

Biclonal gammopathies manifestations (BGMs) are defined as a clonal proliferation of plasma cells or B-lymphoid progenitors that produces 2 different monoclonal proteins known as M-proteins or paraproteins. The aim of our study is to report and analyses the epidemiological, biological and clinical features of the cases of biclonal gammopathies diagnosed at the Hassan II University Hospital Center (HUHC), Fez during a period of 10 years (2010-2020). Among the 782 cases of gammopathies diagnosed by immunofixation (IF), 28 were shows biclonal gammopathies (3.5\%), the sex ratio M/F was 1 and the median age was 63 years. The most frequent isotype was $\mathrm{IgG/IgM}$ with a slight predominance of the Kappa light chain. The most common diagnosis was biclonal gammopathy of undetermined significance (BGUS) in 12 patients (42\%), followed by 10 cases of multiple myeloma (MM) (35\%) and 6 cases of lymphoproliferative syndrome $(21 \%)$. Although the clinical features are similar to monoclonal gammopathy, dentification of BGMs increases diagnostic precision in our region, in particular for multiple myeloma cases and lymphoproliferative syndrome compared to other studies.

Keywords: Biclonal gammopathies, monoclonal gammopathies, multiple myeloma, lymphoproliferative syndrome, Morocco.

Copyright (C) 2020 The Author(s): This is an open-access article distributed under the terms of the Creative Commons Attribution 4.0 International License (CC BY-NC 4.0) which permits unrestricted use, distribution, and reproduction in any medium for non-commercial use provided the original author and source are credited.

\section{INTRODUCTION}

Biclonal gammopathy (BG) is a pathology characterized by the proliferation of one or two clones of lymphocyte resulting in the appearance of 2 abnormal immunoglobulins in excess in the serum called "M" proteins or paraproteins. The biclonal gammopathy may occur with two separate distinct bands on the serum protein electrophoresis or a single band that resolves into two distinct bands on the serum immunofixation. Although the production of paraproteins is characteristically related to the dyscrasias of plasma cells, it can be associated with other neoplasms, especially the B lineage lymphoid, and to a lesser degree with other blood components and solid organ cells.

Biclonal gammopathy is a rare condition with an incidence ranging from 1 to $5 \%$ of the patients presenting clonal plasma-cell proliferations [1-3].

In this study we report the epidemiological, biological, etiological and immunochemical aspect of the biclonal gammopathies diagnosed at the HUHC of $\mathrm{Fez}$ in Morocco over a period of 10 years.

\section{MATERIAL AND METHODS}

This is a retrospective study that included all cases of biclonal gammopathies diagnosed at the biochemistry laboratory of the HUHC between January 2010 and January 2020 by the presence of two monoclonal bands on serum immunofixation. We study the records of the biochemistry department immunofixation registry for identifying patients with biclonal gammopathies from all departments of HUHC since 2010 .

Serum protein electrophoresis (SPE) was performed on a semi-automated device (HYDRASYS; SEBIA®) using agarose gel. The concentration of circulating immunoglobulins $(\mathrm{G}, \mathrm{A}$ and $\mathrm{M})$ was measured by immunoturbidimetry on ARCHITECT® $\mathrm{C} 8000$, the monoclonal component was not quantified. 
To identify the type of immunoglobulin and the Bence-Jones protein, we used the immunofixation technique. Serum immunofixation was performed manually on a blood sample on a dry tube according to the SEBIA $®$ protocol on agarose gel using antisera monospecific for the heavy and light chains of human immunoglobulins (anti- $\gamma$, anti- $\mu$, anti- $\alpha$, anti- $\kappa$, anti- $\lambda$ ). Urinary immunofixation was performed on a 24-hour urine sample using the same serum immunofixation protocol with different free and bound light chain antisera.

We recovered the epidemiological data, such as age and sex, and information on the underlying disease and/or associated diseases, the type of paraprotein, the quantification of immunoglobulins had undergone from the medical records review. Lastly, we analyzed the clinical evolution of patients.

\section{RESULTS}

\section{Patients Characteristics and Associated Diseases}

Between 2010 and 2019, a total of 782 patients with gammopathies were studied, 28 of whom corresponded to the diagnosis of biclonal gammopathy, which represents $3,5 \%$ of the total.

The mean age at diagnosis of these 28 patients was $63 \pm 14$, and 14 of them $(50 \%)$ were men.

The most common diagnosis was biclonal gammopathy of undetermined significance (BGUS) in 12 patients $(42 \%)$. A total of 10 cases $(35 \%)$ were diagnosed as multiple myeloma (MM). Lymphoproliferative syndrome was reported in 6 cases (21\%) with 2 cases of renal amyloidosis, and 1 case for each of the following diseases: chronic lymphocytic leukemia, non-Hodgkin's lymphoma, Waldenström's disease and acute leukemia.

Patients diagnosed with BGUS don't show any clinical signs or lesions of bone or kidney disease or anemia (in favor of multiple myeloma). Only the presence of biclonal spike on serum immunofixation with a serum immunoglobulin level of less than $30 \mathrm{~g} / \mathrm{L}$ and sternal puncture with medullary plasma cells < $10 \%$.

In our series, furthers investigations among patients with BGUS shows renal insufficiency in 3 patients which were related to a glomerulonephritis or nephrotic syndrome, while each vasculitis, systemic joint involvement and headache with altered general condition were reported in 1 patient.

Patients with multiple myeloma, reported bone pain in $90 \%$ of the cases associated with anemic syndrome $(70 \%)$, kidney damage $(50 \%)$, hypercalcemia $(30 \%)$ and neurological damage $(30 \%)$ consisting of tingling and hypoesthesia.

\section{Serum electrophoresis, immunofixation, and other laboratory test findings}

In serum protein electrophoresis monoclonal peak was present in the gamma globulin fraction in 15 cases (Figure-1), while 6 cases had a biclonal peak in the gamma globulin fraction (Figure-2). The remaining cases presented separately a beta 2 globulin peak in 2 cases, one case of a beta 2 globulin peak associated with another gamma globulin peak, one case of hypogammaglobulinemia and the last two cases presented an inflammatory syndrome.

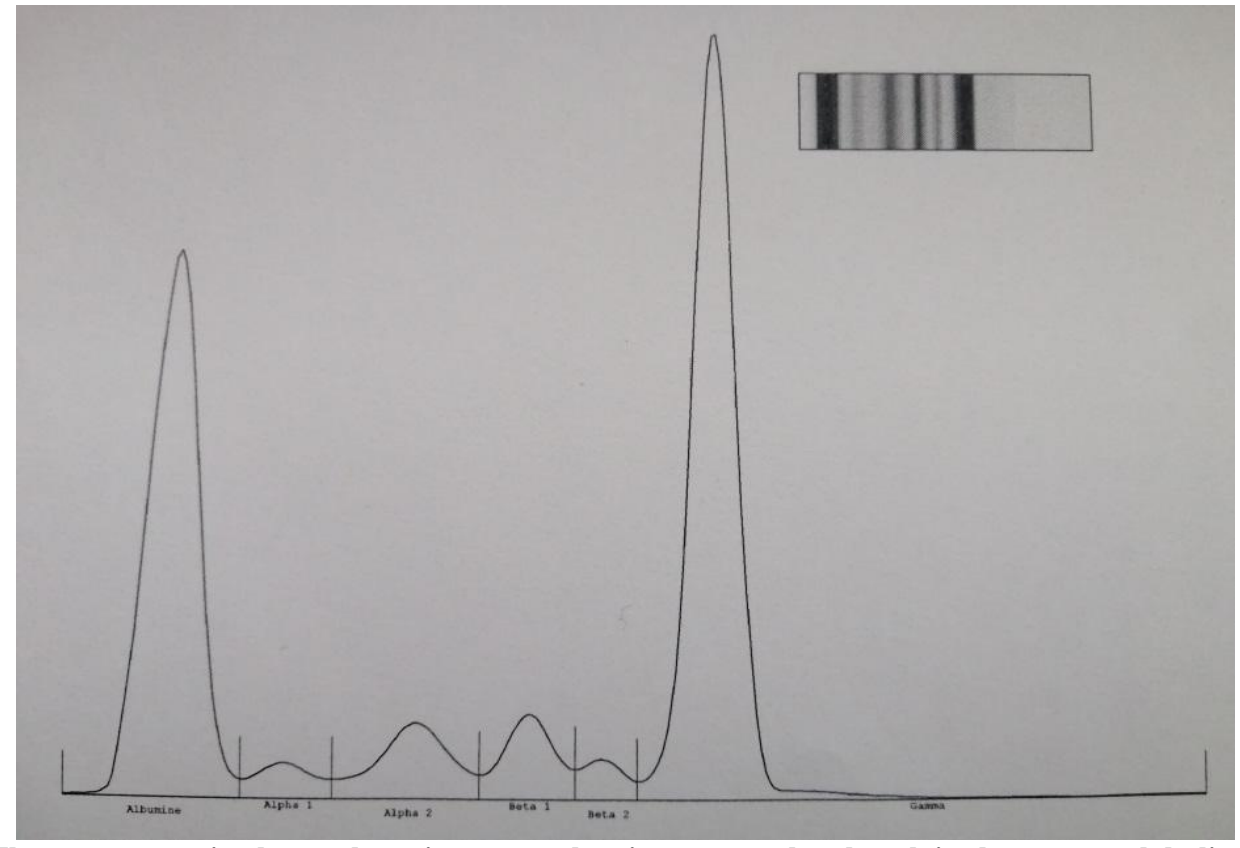

Fig-1: The serum protein electrophoresis pattern showing a monoclonal peak in the gamma globulin fraction 

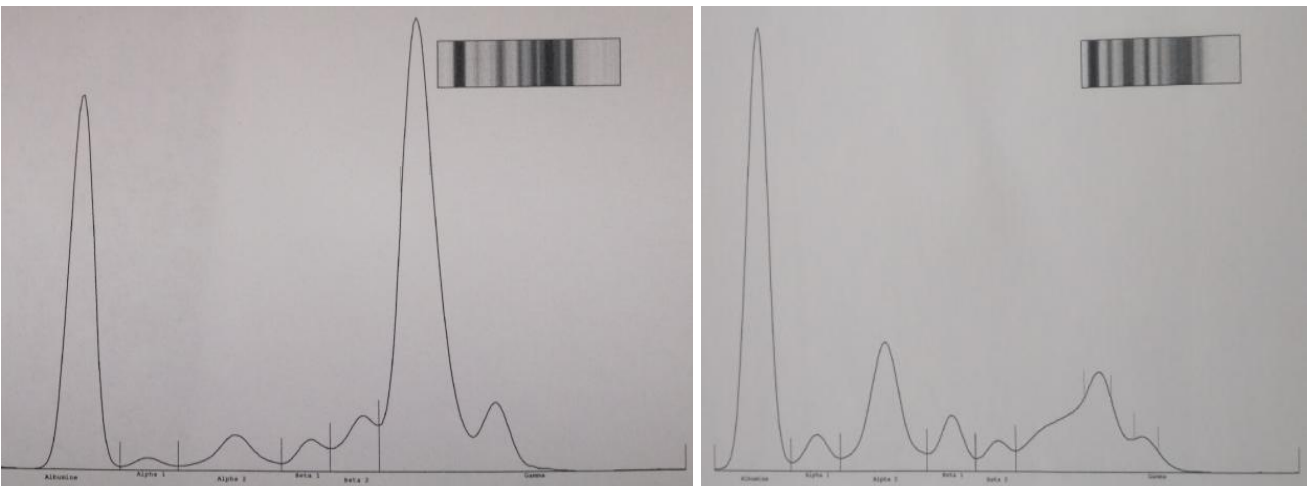

Fig-2: Two serum protein electrophoresis patterns showing a biclonal peak in the gamma globulin fraction

On serum immunofixation, 16 patients had two monoclonal bands with the most common isotype being IgG/IgM ( 8 cases- $50 \%)$ followed by $\operatorname{IgG} / \operatorname{IgA}(5$ cases$31 \%)$, IgG/IgG (2 cases- $12.5 \%$ ) and $\operatorname{IgA} / \operatorname{IgA}$ (1 case$6 \%$ ) with a slight predominance of the Kappa light chain $(55 \%)$.
For the other 12 patients, they presented a monoclonal band associated with a free light chain with a predominance of $\mathrm{IgG} \mathrm{L/L} \mathrm{(5} \mathrm{cases} \mathrm{-} 41 \%$ ) followed by IgG $\mathrm{K} / \mathrm{K}(2$ cases $-16 \%)$, IgA L/K(1 case - $8 \%)$, IgG $\mathrm{K} / \mathrm{L}$ (1 case $-8 \%), \operatorname{IgA~K/K}(1$ case $-8 \%), \operatorname{IgA} \mathrm{L} / \mathrm{L}(1$ case $-8 \%)$ and $\operatorname{IgM~K} / \mathrm{K}(1$ case $-8 \%)$.

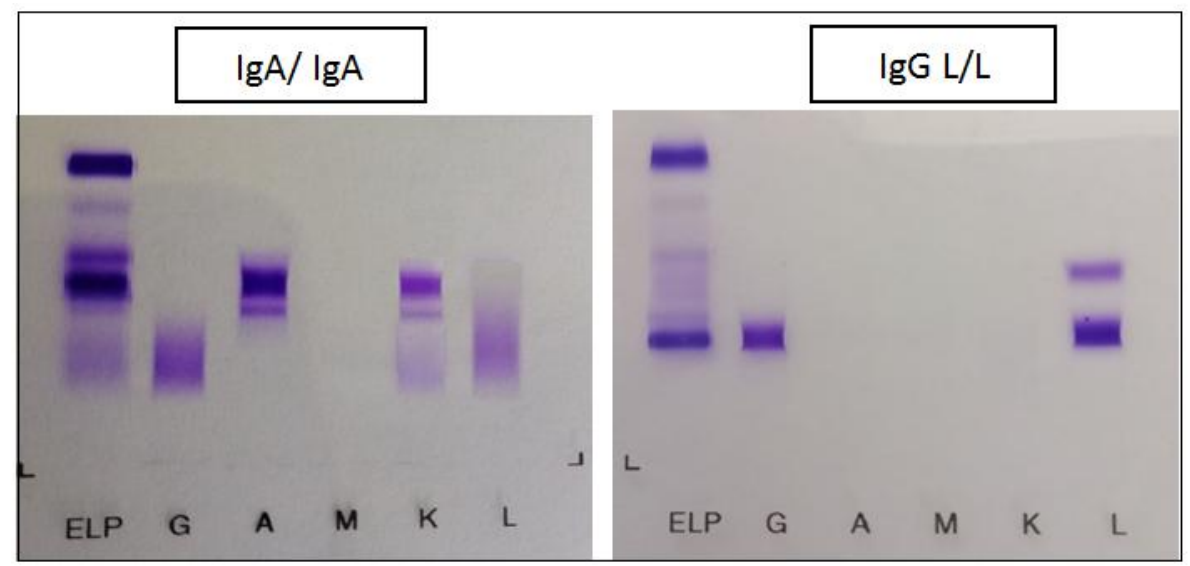

Fig-3: Serum immunofixation of 2 of our patients showing an example of two monoclonal bands (IgA/IgA) and one monoclonal band associated with a light band (IgG L/L)

Urinary immunofixation was performed in 21/28 patients. Bence Jones protein (BJP) was observed in 13 patients $(61 \%)$. The BJP was Lambda in 6 patients and Kappa in 7 patients.
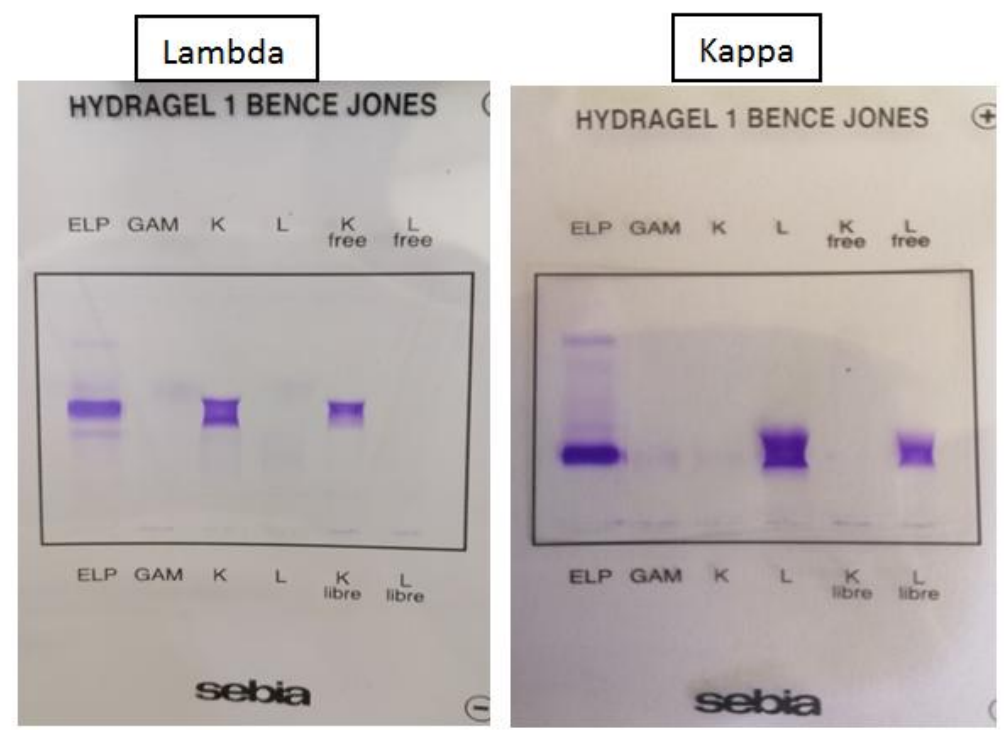

Fig-4: Two urinary immunofixation displaying a positive BJP of lambda and kappa 
Immunoglobulin dosage in the serum was performed in 19 patients; 13 had a single dominant elevated immunoglobulin; 7 had IgG immunoglobulin; 4 had $\operatorname{IgA}$ and 2 had $\operatorname{IgM}$. The mean plasma concentration of $\mathrm{IgG}$ was $19 \mathrm{~g} / \mathrm{l}$ with extremes ranging from 3.2 to $43 \mathrm{~g} / \mathrm{l}$. As for the plasma concentration of $\operatorname{IgA}$ and $\mathrm{IgM}$, the median was $6.8 \mathrm{~g} / \mathrm{l}(0.21$ to $37 \mathrm{~g} / \mathrm{l})$ and $1.5 \mathrm{~g} / 1(0.25$ to $21 \mathrm{~g} / \mathrm{l})$, respectively (Table- 1$)$. The highest immunoglobulin was consistent with the band found during serum immunofixation for the group of patients with a monoclonal band associated with a free light chain. Moreover, it helped to find the dominant band in patients with two monoclonal bands on their serum immunofixation.
The light chain assay in the serum was performed in 3 patients whose ratio (Kappa/Lambda) was pathological. This ratio was low in 1 patient with excess in Lambda light chains and high in 2 patients with Kappa light chains.

Patients were followed up clinically and biologically from 6 months to 8 years with a mean of 40 months. Patients with 2 monoclonal bands lost one band in the control in $31 \%$ of cases; this remaining band was associated with the elevated immunoglobulin on the serum dosage.

Table-1: Epidemiological and immuno-biochemical characteristics of biclonal gammopathies

\begin{tabular}{|c|c|c|c|c|c|c|c|c|c|}
\hline & Sex & Age & Paraprotein & & diagnosis & BJP & IgG $(g / \mathbf{l})$ & $\operatorname{IgM}(\mathrm{g} / \mathrm{l})$ & $\operatorname{IgA}(\mathrm{g} / \mathrm{l})$ \\
\hline 1 & $F$ & 60 & IgG Kappa & IgM kappa & BGUS & Negatif & 17 & 21 & 2 \\
\hline 2 & $\mathrm{M}$ & 32 & IgG kappa & IgA lambda & Renal amyloidosis & Negatif & & & \\
\hline 3 & $\mathrm{M}$ & 53 & IgG kappa & IgA kappa & multiple myeloma & kappa & & & \\
\hline 4 & $\mathrm{M}$ & 71 & IgG Kappa & IgM lambda & BGUS & Negatif & 3,55 & 0,25 & 0,25 \\
\hline 5 & $\mathrm{~F}$ & 69 & IgG Lambda & IgM lambda & LMNH & & 4,69 & 0,05 & 0,21 \\
\hline 6 & $\mathrm{~F}$ & 70 & IgG kappa & IgM kappa & BGUS & Kappa & 8,38 & 0,56 & 1,45 \\
\hline 7 & $\mathrm{M}$ & 61 & IgG Lambda & IgM Kappa & BGUS & & & & \\
\hline 8 & $\mathrm{M}$ & 82 & IgG kappa & IgG lambda & BGUS & kappa & 27 & 1,14 & 1,99 \\
\hline 9 & $\mathrm{M}$ & 77 & IgG lambda & IgM kappa & Waldenström & Negatif & 5,78 & 20 & 0,35 \\
\hline 10 & $\mathrm{~F}$ & 38 & IgG kappa & IgM lambda & BGUS & & & & \\
\hline 11 & $\mathrm{M}$ & 65 & IgA kappa & IgG lambda & BGUS & lambda & 43 & 0,27 & 3,65 \\
\hline 12 & $\mathrm{~F}$ & 65 & IgA kappa & IgG kappa & BGUS & Negatif & 4,59 & 0,46 & 35 \\
\hline 13 & $\mathrm{~F}$ & 80 & IgA lambda & IgG lambda & BGUS & & 5,94 & 0,25 & 21,89 \\
\hline 14 & $\mathrm{M}$ & 39 & IgA kappa & IgA kappa & multiple myeloma & kappa & 3,2 & 0,48 & 37,15 \\
\hline 15 & $\mathrm{~F}$ & 39 & IgG Kappa & IgG Kappa & multiple myeloma & Kappa & 42,46 & 0,76 & 0,25 \\
\hline 16 & $\mathrm{~F}$ & 78 & IgG lambda & IgM kappa & LLC & Negatif & 17,15 & 1,53 & 0,51 \\
\hline 17 & $\mathrm{M}$ & 69 & IgG kappa & lambda & Acute leukemia & & & & \\
\hline 18 & $\mathrm{M}$ & 65 & IgA lambda & Kappa & multiple myeloma & lambda & & & \\
\hline 19 & M & 70 & IgG kappa & Kappa & multiple myeloma & Kappa & & & \\
\hline 20 & $\mathrm{~F}$ & 67 & IgA lambda & lambda & BGUS & Negatif & & & \\
\hline 21 & $\mathrm{~F}$ & 79 & IgG lambda & lambda & multiple myeloma & lambda & 40,74 & 0,27 & 0,25 \\
\hline 22 & $\mathrm{M}$ & 62 & IgM kappa & kappa & Renal amyloidosis & Negatif & & & \\
\hline 23 & F & 51 & IgA kappa & kappa & multiple myeloma & & 13,66 & 0,98 & 17,7 \\
\hline 24 & $\mathrm{~F}$ & 90 & IgG lambda & lambda & multiple myeloma & lambda & 35,02 & 0,3 & 0,45 \\
\hline 25 & $\mathrm{M}$ & 60 & IgG lambda & lambda & multiple myeloma & lambda & 40,3 & 0,25 & 0,6 \\
\hline 26 & $\mathrm{M}$ & 71 & IgG lambda & lambda & BGUS & & 3,25 & 0,48 & 0,25 \\
\hline 27 & $\mathrm{~F}$ & 50 & IgG lambda & lambda & multiple myeloma & lambda & 42,46 & 0,48 & 0,25 \\
\hline 28 & $\mathrm{~F}$ & 61 & IgG Kappa & kappa & BGUS & kappa & 11,7 & 0,25 & 0,53 \\
\hline
\end{tabular}

\section{DISCUSSION}

Biclonal gammopathies are a group of diseases characterized by the clonal proliferation of plasma cells or their B-lymphoid progenitors and is associated with the production of 2 abnormal immunoglobulins known as M proteins or paraproteins [4].

The diagnosis of biclonal gammopathy involves determining the presence of 2 different electrophoretic bands, which can be composed of equal or different combinations of heavy and light chains. The production of these paraproteins may be due to the existence of a single clone of B-lymphoid cells, which subsequently diversifies into 2 independent clones after a process of antigenic selection $[4,5]$. Or is instead due to the presence of 2 different clones at the start of the tumor transformation [6].

During our 10-year study, 1579 serum immunofixations were performed, of which 782 concluded to have gammopathy with 28 cases of biclonal gammopathies, indicating an incidence of $3.5 \%$ of all gammopathies. This rate is comparable to that reported in the literature with an incidence ranging from $1 \%$ to $5 \%$ including a Tunisian study they found an incidence of $2 \%$ BG (6 cases out of 288 cases) [1-3]. 
The mean age of the patients in our series at the time of diagnosis was 63 years, indicating that gammopathies are diseases of the elderly subject. This median age is comparable to that reported by Garcia et al., but remains lower than that described by Leroy et al., (72 years) $[3,7]$. There was no sex predominance in our series unlike the different series in the literature which indicates a male predominance [1-3].

On serum protein electrophoresis, $17 \%$ of the patients had a biclonal band while $53 \%$ had a monoclonal band. These results are comparable to those reported in literature $[1,8]$.

Our findings indicate that $30 \%$ of the patients did not present a peak in the gamma globulin zone. This result attests the insufficient sensitivity of the SPE for the detection of biclonal gammopathies, especially those with low serum monoclonal Ig levels. This finding reveals the interest in requesting serum immunofixation in the event of suspicion of gammopathies or diagnostic doubt [9].

The most common composition of Ig in our series was the $\operatorname{IgG/IgM~combination~}(50 \%)$, which is similar to the findings in the series by Leroy et al., [3] and Riddle et al., [10]. But different from those observed in the series by Garcia et al., [7] (IgG/IgG) and in the series of Nilsson et al., [11] (IgG/IgG), of Perkins et al., [12] (IgA/IgA) and of Kyle et al., [1] (IgG/IgA). The differences in composition of the gammopathies in the 7 series cannot be explained by epidemiolocal data or by associated diseases. The differences could be due to the different levels of sensitivity of the paraprotein detection technique employed for each series or to the small number of patients enrolled in each series.

In our series, there is a slight predominance of kappa light chains in about $55 \%$ of the BG cases revealed by the two monoclonal IF bands. The result is similar to that found in the specific biclonal series published in literature $(55 \%-66 \%)[1,11]$.

In the literature several studies have excluded monoclonal bands associated with a free light chain from the study of biclonal gammopathies because one of the two light bands observed is an excess that migrates faster than the light chains associated with heavy chains. This could be due to the asynchronous production of the components of immunoglobulin molecules, leading to the synthesis of an intact monoclonal band plus an excess of monoclonal free light chains [13].

Urinary immunofixation in our study revealed the presence of BJP in $61 \%$ of cases; whereas in the study by Garcia et al., [7], it was positive in only $14.8 \%$. These differences may be due to a higher incidence of multiple myeloma in our study because BJP was positive in $55 \%$ of the multiple myeloma group compared to $22 \%$ of the BGUS group in the study done by Kyle et al., [1].

Table-2: Comparison of etiological diagnoses of biclonal gammopathies their isotype distributions and prevalence of the Kappa light chains in the different studies carried out from 1981 to 2016 and our study

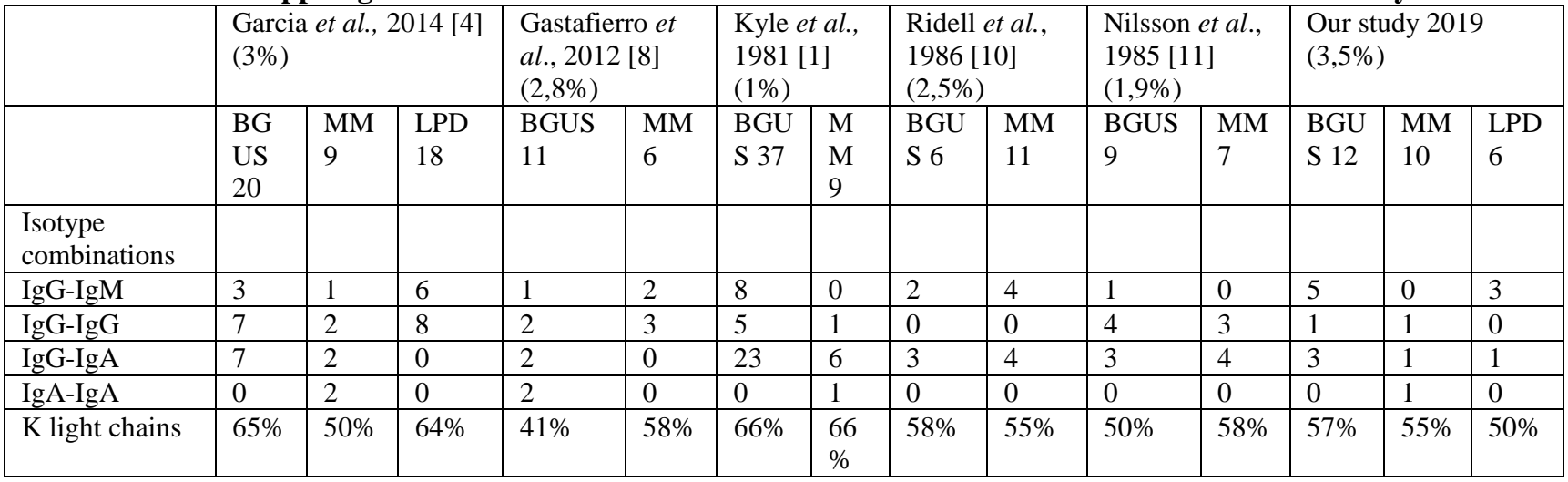

The BGUS and lymphoproliferative syndrome are generally the most common etiological diagnosis found in published BG series, which is consistent with our study (Table-2).

Given the lack of studies BG in the Maghreb region, we rely the Tunisian monoclonal gammopathy study done by Mseddi-Hdiji [2] which has a higher rate of multiple myeloma than of MGUS compared to the various other studies. On the other hand, we can also explain the rate of multiple myeloma by a delay in the discovery of BGUS which, as various studies have shown, can progress to multiple myeloma after years of asymptomatic progression [14].

In the follow-up of these cases, it is interesting to note that one of the $\mathrm{M}$ components has decreased or disappeared in 5 of the 16 patients $(31 \%)$. Two of them who suffered from multiple myeloma lost one of the $\mathrm{M}$ components after receiving chemotherapy, one of them had waldenström disease (IgG-IgM) losing the IgG component after chemotherapy, the two patients left 
had BGUS and received corticosteroid therapy for their respectively renal and neurological diseases. Previous reports documenting this finding suggest that the disappearing bands obeyed regulatory mechanisms or that in neoplastic cell lines additional mutations had occurred [15]. In the study by Garcia et al., [7] and Nilsson et al., [11], the disappearance of a monoclonal band was found in $38 \%$ and $30 \%$ of their cases respectively.

According to the various studies in the literature, the plasma level of immunoglobulins is often elevated due to the lymphocyte proliferation in the BG. Our study has found an increase in a single immunoglobulin of which $36 \%$ were $\operatorname{IgG}$ vs $32 \%$ according to the study of Riddel et al., [10], 10\% were $\operatorname{IgM}$ vs $28 \%$ and $21 \%$ were $\operatorname{IgA}$ vs $28 \%$. Immunoglobulin levels were higher than $20 \mathrm{~g} / \mathrm{l}$ in $52 \%$ of cases compared to $28 \%$ in Riddle et al., [10] and $23 \%$ in Garcia et al., [7].

Immunoglobulin is used as a tumor marker for the recognition of the clinical disorder and for monitoring the progression, response or relapse of the disease, but also at the time of diagnosis to point out the dominant immunoglobulin since in the literature it is found that the dominant clone is the main actor in the evolution of the disease [14].

\section{CONCLUSION}

This is the first contribution for the study of BG in the Fez-Meknes region, Morocco. We reported here a biclonal gammopathies case series from the Hassan II University Hospital Center, Fez. BG is a rather rare form of gammopathies with a prevalence of only $3,5 \%$ of all cases of our series. The most common diagnosis was respectively BGUS (42\%), MM (35\%) and LPS $(21 \%)$. These results are comparable of the literature and more study must be conducted to detect an eventual specificity of regional or national population.

\section{REFERENCS}

1. Kyle, R. A., Robinson, R. A., \& Katzmann, J. A. (1981). The clinical aspects of biclonal gammopathies: review of 57 cases. The American journal of medicine, 71(6), 999-1008.

2. Mseddi-Hdiji, S., Haddouk, S., Ayed, M. B., Tahri, N., Elloumi, M., Baklouti, S., ... \& Masmoudi, H. (2005). Gammapathies monoclonales en Tunisie: analyse épidémiologique, immunochimique et étiologique d'une série de 288 cas. Pathologie Biologie, 53(1), 19-25.

3. Leroy, H., Decaux, O., Ianotto, J. C., Guenet, L., Ruelland, A., Sebillot, M., ... \& Grosbois, B. (2008). Caractéristiques cliniques et biologiques des gammapathies biclonales. Description d'une cohorte de 203 patients. La Revue de médecine interne, (29), S320.
4. Lichtman, M. A., Kaushansky, K., Beutler E., Kipps, T. J, Seligsohn, U., Prchal, J. T. (2010). Essential monoclonal gammopathies. s.l. : Williams haematology. 8th ed. McGraw-Hill. 1638-1644.

5. Tawfik, N. M., El Deeb, M., \& Nasr, A. S. (2013). Monoclonal gammopathy among patients with chronic hepatitis $\mathrm{C}$ virus infection. The American journal of the medical sciences, 345(5), 366-368.

6. Brito-Zerón, P., Retamozo, S., Gandía, M., Akasbi, M., Pérez-De-Lis, M., Diaz-Lagares, C., ... \& Sisó, A. (2012). Monoclonal gammopathy related to Sjögren syndrome: a key marker of disease prognosis and outcomes. Journal of autoimmunity, 39(1-2), 43-48.

7. García-García, P., Enciso-Alvarez, K., DiazEspada, F., Vargas-Nuñez, J. A., Moraru, M., \& Yebra-Bango, M. (2015). Biclonal gammopathies: retrospective study of 47 patients. Revista Clínica Española (English Edition), 215(1), 18-24.

8. Guastafierro, S., Ferrara, M. G., Sica, A., Parascandola, R. R., Santangelo, S., \& Falcone, U. (2012). Serum double monoclonal components and hematological malignancies: only a casual association? Review of 34 cases. Leukemia Research, 36(10), 1274-1277.

9. Katzmann, J., Kyle, R. A., Lust, J., Snyder, M., \& Dispenzieri, A. (2012). Immunoglobulins and Laboratory Recognition of Monoclonal Proteins. s.1: Neoplastic Diseases of the Blood, 565-588.

10. Riddell, S. T. A. N. L. E. Y., Traczyk, Z. D. Z. I. S. T. A. W. A., Paraskevas, F. R. I. X. O. S., \& Israels, L. G. (1986). The double gammopathies. Clinical and immunological studies. Medicine, 65(3), 135-142.

11. Nilsson, T., Norberg, B., Rudolph!, O., \& Jacobsson, L. (1986). Double gammopathies: incidence and clinical course of 20 patients. Scandinavian journal of haematology, 36(1), 103-106.

12. Perkins, S. L., \& Ooi, D. S. (1987). A Study of Biclonal Gammopathies. Clinical biochemistry. 20(4).

13. Srinivasan, V. K., Bhagat, P., Bansal, F., \& Chhabra, S. (2016). Occurrence of double monoclonal bands on protein electrophoresis: an unusual finding. Indian Journal of Hematology and Blood Transfusion, 32(1), 184-188.

14. Mullikin, T. C., Rajkumar, S. V., Dispenzieri, A., Buadi, F. K., Lacy, M. Q., Lin, Y., ... \& Russell, S. J. (2016). Clinical characteristics and outcomes in biclonal gammopathies. American journal of hematology, 91(5), 473-475.

15. Bast, E. J. E. G., Slaper- Cortenbach, C. M., Verdonck, L. F., Samson, W., \& Ballieux, R. E. (1985). Transient expression of a second monoclonal component in two forms of biclonal gammopathy. British journal of haematology, 60(1), 91-97. 